\title{
Bäcklund Transformations for the Trigonometric Gaudin Magnet ${ }^{\star}$
}

\author{
Orlando RAGNISCO and Federico ZULLO
}

Dipartimento di Fisica Universitá Roma Tre and Istituto Nazionale di Fisica Nucleare, Sezione di Roma, I-00146 Roma, Italy

E-mail: ragnisco@fis.uniroma3.it, zullo@fis.uniroma3.it

URL: http://webusers.fis. uniroma3/ ragnisco/

Received December 12, 2009, in final form January 27, 2010; Published online January 29, 2010 doi:10.3842/SIGMA.2010.012

\begin{abstract}
We construct a Bäcklund transformation for the trigonometric classical Gaudin magnet starting from the Lax representation of the model. The Darboux dressing matrix obtained depends just on one set of variables because of the so-called spectrality property introduced by E. Sklyanin and V. Kuznetsov. In the end we mention some possibly interesting open problems.
\end{abstract}

Key words: Bäcklund transformations; integrable maps; Gaudin systems

2010 Mathematics Subject Classification: 37J35; 70H06; 70H15

\section{Introduction}

Bäcklund transformations are a prominent tool in the theory of integrable systems and soliton theory. Historically they appeared first in the works of Bianchi [1] and Bäcklund [2] on surfaces of constant curvature and allowed them to pass from a surface of constant curvature to a new one, or from a solution of a given PDE to a new one. By this point of view Bäcklund transformations have been extensively exploited $[3,4,5,6]$. In the field of finite-dimensional systems they can be seen as integrable Poisson maps that discretize a family of continuous flows; one of the earliest account of this subject is in [7] where the term integrable Lagrange correspondences is used for integrable maps. This point of view has been widely explored by Suris [8], Sklyanin [9], Sklyanin and Kuznetsov [10], Kuznetsov and Vanhaecke [11]. Numerous relevant results appeared in the 90 's and at the beginning of the present century on exact time discretizations of many body systems. Our paper is an ideal continuation, almost 10 years later, of a joint paper by our dear friend Vadim, Andy Hone and O.R. [12], where the same problem has been studied and solved for the rational Gaudin chain. The key observation we make (see also [13]) is that the trigonometric Gaudin model with $N$ sites is just the rational Gaudin model with $2 N$ sites with an extra reflection symmetry ("inner automorphism"), entailing the following involution on the corresponding Lax matrix:

$$
L(z)=\sigma_{3} L(-z) \sigma_{3}
$$

where $z$ is the spectral parameter, and $\sigma_{3}$ is the usual Pauli matrix diag $(1,-1)$. In the following section we will derive (1) from the standard form of the trigonometric Lax matrix. Here we can already argue that, to preserve the reflection symmetry, the elementary dressing matrix, that we will call $D$ after Darboux, has to enjoy a similar property (up to an inessential scalar

${ }^{\star}$ This paper is a contribution to the Proceedings of the XVIIIth International Colloquium on Integrable Systems and Quantum Symmetries (June 18-20, 2009, Prague, Czech Republic). The full collection is available at http://www.emis.de/journals/SIGMA/ISQS2009.html 
factor), and consequently it has to exhibit pairs of singular points in the spectral complex plane. Those singular points can be (opposite) zeroes and/or (opposite) poles, due to the symmetric role played by $D$ and $D^{-1}$. As the Bäcklund transformation between the "old" Lax matrix $L$ and the updated $\tilde{L}$ has to preserve the spectral invariants of $L$, it has to be defined through a similarity map:

$$
\tilde{L}(z)=D(z) L(z)[D(z)]^{-1} .
$$

Obviously we should require that the rational structure of the Lax matrix be preserved, i.e. that the updated matrix has the same number of poles and zeroes as the old one. In the sequel we will focus our attention on elementary Bäcklund transformations, where the corresponding $D$ has just one pair of (opposite) singular points.

\section{The trigonometric Gaudin magnet}

As it is well known the trigonometric Gaudin model is governed by the following Lax matrix:

$$
\begin{aligned}
& L(\lambda)=\left(\begin{array}{cc}
A(\lambda) & B(\lambda) \\
C(\lambda) & -A(\lambda)
\end{array}\right), \\
& A(\lambda)=\sum_{j=1}^{N} \cot \left(\lambda-\lambda_{j}\right) s_{j}^{3}, \quad B(\lambda)=\sum_{j=1}^{N} \frac{s_{j}^{-}}{\sin \left(\lambda-\lambda_{j}\right)}, \quad C(\lambda)=\sum_{j=1}^{N} \frac{s_{j}^{+}}{\sin \left(\lambda-\lambda_{j}\right)} .
\end{aligned}
$$

The dynamical variables $\left(s_{j}^{+}, s_{j}^{-}, s_{j}^{3}\right), j=1, \ldots, N$, obey to the Poisson structure given by the brackets:

$$
\left\{s_{j}^{3}, s_{j}^{ \pm}\right\}=\mp i \delta_{j k} s_{k}^{ \pm}, \quad\left\{s_{j}^{+}, s_{j}^{-}\right\}=-2 i \delta_{j k} s_{k}^{3},
$$

with the $N$ Casimirs given by

$$
s_{j}^{2}=\left(s_{j}^{3}\right)^{2}+s_{j}^{+} s_{j}^{-} .
$$

This structure corresponds to the trigonometric $r_{t}$ matrix, given by

$$
r_{t}(\lambda)=\frac{1}{\sin (\lambda)}\left(\begin{array}{cccc}
\cos (\lambda) & 0 & 0 & 0 \\
0 & 0 & 1 & 0 \\
0 & 1 & 0 & 0 \\
0 & 0 & 0 & \cos (\lambda)
\end{array}\right)
$$

with the Lax matrix satisfying the linear r-matrix Poisson algebra,

$$
\left\{L^{1}(\lambda), L^{2}(\mu)\right\}=\left[r_{t}(\lambda-\mu), L^{1}(\lambda)+L^{2}(\mu)\right]
$$

where, as usually, the superscripts on the matrices denote tensor products:

$$
L^{1}=L \otimes I, \quad L^{2}=I \otimes L .
$$

The equation (5) is equivalent to the following Poisson brackets for the elements $A(u), B(u)$ and $C(u)$ :

$$
\begin{aligned}
& \{A(\lambda), A(\mu)\}=\{B(\lambda), B(\mu)\}=\{C(\lambda), C(\mu)\}=0, \\
& \{A(\lambda), B(\mu)\}=\frac{\cos (\lambda-\mu) B(\mu)-B(\lambda)}{\sin (\lambda-\mu)}
\end{aligned}
$$




$$
\begin{aligned}
& \{A(\lambda), C(\mu)\}=\frac{C(\lambda)-\cos (\lambda-\mu) C(\mu)}{\sin (\lambda-\mu)}, \\
& \{B(\lambda), C(\mu)\}=\frac{2(A(\mu)-A(\lambda))}{\sin (\lambda-\mu)} .
\end{aligned}
$$

Through the "uniformization" mapping:

$$
\lambda \rightarrow z=e^{i \lambda}
$$

the Lax matrix (3) acquires a rational form in $z$ :

$$
-i L(z)=\sum_{j=1}^{N} s_{j}^{3} \sigma_{3}+\sum_{j=1}^{N}\left(\frac{L_{1}^{j}}{z-z_{j}}-\sigma_{3} \frac{L_{1}^{j}}{z+z_{j}} \sigma_{3}\right),
$$

where the matrices $L_{1}^{j}, j=1, \ldots, N$, have the simple form:

$$
L_{1}^{j}=z_{j}\left(\begin{array}{cc}
s_{j}^{3} & s_{j}^{-} \\
s_{j}^{+} & -s_{j}^{3}
\end{array}\right) .
$$

The equation (6) leads to the reflection symmetry (1):

$$
L(z)=\sigma_{3} L(-z) \sigma_{3}
$$

\section{The Darboux matrix}

The simplest choice for the spectral structure of the Darboux-dressing matrix requires that it obeys the reflection symmetry (1) and contains only one pair of opposite simple poles. Then, it reads:

$$
D=D_{\infty}+\frac{D_{1}}{z-\xi}-\frac{\sigma_{3} D_{1} \sigma_{3}}{z+\xi} .
$$

The matrix $D_{\infty}$, i.e. $\lim _{z \rightarrow \infty} D(z)$ defines the normalization of the problem. The equation (2), rewritten in the form:

$$
\tilde{L}(z) D(z)=D(z) L(z)
$$

in the limit $z \rightarrow \infty$ yields:

$$
\left(\tilde{S}_{z}\right) \sigma_{3} D_{\infty}=D_{\infty}\left(S_{z}\right) \sigma_{3}
$$

where by $S_{z}$ we have denoted the $z$-component of the total "spin" $S$. As $S_{z}$ Poisson commutes with $\operatorname{tr} L^{2}$, the generating function of the complete family of involutive Hamiltonians, it has to be preserved by our Bäcklund transformation, which is a symmetry for the whole hierarchy. This implies $D_{\infty}$ to be diagonal.

As for bounded values of $z$, equation (8) implies that both sides have equal residues at the simple poles $\pm z_{j}, \pm \xi$. However, in view of the symmetry property (1), (7) it will be enough to look at half of them, say $z_{j}, \xi$. The corresponding equations will be:

$$
\begin{aligned}
& \tilde{L}_{1}^{(j)} D\left(z_{j}\right)=D\left(z_{j}\right) L_{1}^{(j)}, \\
& \tilde{L}(\xi) D_{1}=D_{1} L(\xi) .
\end{aligned}
$$


The crucial problem to solve now is to ensure that (9), (10) provide an explicit (and symplectic) mapping between the old and the new spin variables. In other words, to get a Darboux matrix that depends just on one set of variables, say the old ones. As it has been shown for instance in $[10,12]$, this can be done thanks to the so-called spectrality property. In the present context, this amounts to require that $\operatorname{det} D$ possess, in addition to the two opposite poles $\pm \xi$, two opposite nondynamical zeroes, say $\pm \eta$ and that $D_{1}$ is, up to a factor, a projector. Again, by symmetry it will be enough to look at one of the zeroes, say $\eta$. By setting $z=\eta$ in (8) we get

$$
\tilde{L}(\eta) D(\eta)=D(\eta) L(\eta)
$$

But $D(\eta)$ is a rank one matrix, having a one dimensional Kernel $|K(\eta)\rangle$, whence:

$$
0=D(\eta) L(\eta)|K(\eta)\rangle
$$

entailing

$$
L(\eta)|K(\eta)\rangle=\mu(\eta)|K(\eta)\rangle,
$$

i.e. the points $\pm \eta, \pm \mu(\eta)$ belong to the spectral curve $\operatorname{det}(L(z)-\mu I)=0 .|K(\eta)\rangle$ is then fully determined in terms of the old dynamical variables. The equation (10) give us another one dimensional Kernel $K(\xi)$ because also $D_{1}$ is a rank 1 matrix, so (10) entails:

$$
L(\xi)|\Omega(\xi)\rangle=\mu(\xi)|\Omega(\xi)\rangle .
$$

The two spectrality conditions (11), (12) allow to write $D$ in terms of the old dynamical variables and of the two Bäcklund parameters $\xi$ and $\eta$, so that the Bäcklund equations (9) yield an explicit map between the new (tilded) and the old (untilded) dynamical variables. In order to clarify the point above let us make some observations. First of all note that requiring $D_{1}$ to be a rank one matrix amounts to require that the determinant of $\left(z^{2}-\xi^{2}\right) D(z)$ be zero for $z=\xi$ or, by symmetry, for $z=-\xi$. In fact:

$$
\left.\left(z^{2}-\xi^{2}\right) D(z)\right|_{z=\xi}=2 \xi D_{1},\left.\quad\left(z^{2}-\xi^{2}\right) D(z)\right|_{z=-\xi}=2 \xi \sigma_{3} D_{1} \sigma_{3} .
$$

Since two Darboux matrices differing just by a multiplicative scalar factor define the same BT, we can choose to work with a modified Darboux matrix $D^{\prime}(z)$ defined by the relation:

$$
D^{\prime}(z) \equiv \frac{z^{2}-\xi^{2}}{z} D(z)
$$

Hence, to ensure that the spectrality property holds true we have to require $\operatorname{det} D^{\prime}(z)$ to vanish at $z=\xi$ and $z=\eta$. The form taken by the Darboux matrix $D^{\prime}(z)$ can be further simplified by writing:

$$
D^{\prime}(z)=z^{-1} \hat{A}+\hat{B}+\hat{C} z .
$$

The matrix $\hat{C}$ is immediately seen to be a diagonal one by looking at the behavior for large values of $z$ and requiring $\tilde{S}_{z}=S_{z}$. On the other hand, $L(0)$ as well as its dressed version $\tilde{L}(0)$ are diagonal matrices:

$$
L(0)=\sum_{j=1}^{N} S_{z}^{(j)} \sigma_{3}-\sum_{j=1}^{N} \frac{L_{1}^{(j)}+\sigma_{3} L_{1}^{(j)} \sigma_{3}}{z_{j}} .
$$

This readily implies that $\hat{A}$ in (13) is diagonal. In turn, (1) implies that if even powers of $z$ are diagonal, odd powers must be off-diagonal, entailing that $\hat{B}$ is an off-diagonal matrix. The 
two matrices $\hat{A}$ and $\hat{C}$ are then given respectively by $\operatorname{diag}\left(a_{1}, a_{2}\right)$, $\operatorname{diag}\left(c_{1}, c_{2}\right)$, whereas the off-diagonal matrix $\hat{B}$ is given by $\left(\begin{array}{cc}0 & b_{1} \\ b_{2} & 0\end{array}\right)$.

We get a deeper insight on the parameterization of matrices $\hat{A}, \hat{B}, \hat{C}$ resorting again to the spectrality property: we stress once more that this amounts to requiring $D^{\prime}(\xi)$ and $D^{\prime}(\eta)$ to be rank one matrices. This means that there exists a function of one variable, say $p$, such that:

$$
\begin{aligned}
& \left\{\begin{array}{l}
c_{1} \xi+a_{1} / \xi+b_{1} p(\xi)=0, \\
b_{2}+p(\xi)\left(c_{2} \xi+a_{2} / \xi\right)=0 ;
\end{array}\right. \\
& \left\{\begin{array}{l}
c_{1} \eta+a_{1} / \eta+b_{2} p(\eta)=0, \\
b_{2}+p(\eta)\left(c_{2} \eta+a_{2} / \eta\right)=0 .
\end{array}\right.
\end{aligned}
$$

The four equations (14), (15) leave us with two undetermined parameters, one of which is a global multiplicative factor for $D^{\prime}(z)$, say $\beta$. The other is denoted by $\gamma$. The parameterization of $D^{\prime}$ reads as follows:

$$
D^{\prime}(z)=\beta\left(\begin{array}{cc}
\frac{z(p(\eta) \eta-p(\xi) \xi)}{\gamma}+\frac{(p(\xi) \eta-p(\eta) \xi) \eta \xi}{\gamma z} & \frac{\xi^{2}-\eta^{2}}{\gamma} \\
\frac{\gamma p(\xi) p(\eta)\left(\xi^{2}-\eta^{2}\right)}{\eta \xi} & \frac{\gamma(p(\eta) \eta-p(\xi) \xi)}{z}+\frac{\gamma z(p(\xi) \eta-p(\eta) \xi)}{\eta \xi}
\end{array}\right) .
$$

The kernel of $D(\xi)$ (resp. $D(\eta))$ ) is simply given by the row $|\Omega(\xi)\rangle=(1, p(\xi))^{T}(\operatorname{resp} .|\Omega(\eta)\rangle=$ $\left.(1, p(\eta))^{T}\right)$. It is an eigenvectors of $L(\xi)$ (resp. $L(\eta)$ ). Hence $p(\xi)$ can be written as:

$$
p(\xi)=\frac{\mu(\xi)-A(\xi)}{B(\xi)},
$$

where we recall that $\mu(z)$ is such that $\mu^{2}(z)=A^{2}(z)+B(z) C(z)$ and $A(z), B(z), C(z)$ are given by (4). In terms of $p(\eta), p(\xi)$, the matrices $D_{\infty}$ and $D_{1}$ in (7) take the form:

$$
\begin{aligned}
& D_{\infty}=\beta\left(\begin{array}{cc}
\frac{p(\eta) \eta-p(\xi) \xi}{\gamma} & 0 \\
0 & \gamma \frac{p(\xi) \eta-p(\eta) \xi}{\eta \xi}
\end{array}\right), \\
& D_{1}=\beta\left(\eta^{2}-\xi^{2}\right)\left(\begin{array}{cc}
\frac{p(\xi)}{\gamma} & -\frac{1}{\gamma} \\
-\gamma \frac{p(\xi) p(\eta)}{\eta \xi} & \gamma \frac{p(\eta)}{\eta \xi}
\end{array}\right) .
\end{aligned}
$$

Since the Darboux matrix $D(z)$ is completely known in terms of one set of dynamical variables, equation (9) yields an explicit Bäcklund transformation for the trigonometric Gaudin magnet.

In a forthcoming paper we will prove that (9) provides indeed a symplectic map between old and new dynamical variables, and moreover that, according to a Sklyanin conjecture, the Darboux matrix (13) is in fact identical to Lax matrix of the elementary trigonometric Heisenberg magnet. The interpolating Hamiltonian flow will be also derived and some examples of discrete dynamics will be displayed and discussed.

\section{Acknowledgments}

This paper is intended to be a contribution to the Proceedings of the International Conference "Integrable Systems and Quantum Symmetries 2009", organized by Professor C. Burdík and held in Prague, June 18-20, 2009. One of the authors (O.R.) wants to warmly thank for his hospitality the Newton Institute for Mathematical Sciences, and all the organizers and the participants to the Program "Discrete Integrable Systems". It was in fact during his stay in Cambridge that the main ideas presented in the paper have been made precise. Also, he acknowledges enlightening discussions with A. Levine (ITEF) at the workshop "Einstein at SISSA 2009", partially funded by the Russian Foundation for Basic Research within the project "The Theory of Nonlinear Integrable Systems". 


\section{References}

[1] Bianchi L., Ricerche sulle superficie elicoidali e sulle superficie a curvatura costante, Ann. Sc. Norm. Super. Pisa Cl. Sci. (1) 2 (1879), 285-341.

[2] Bäcklund A.V., Einiges über Curven- und Flächen-Transformationen, Lunds Univ. Årsskr. 10 (1874), 1-12.

[3] Rogers C., Bäcklund transformations in soliton theory, in Soliton Theory: a Survey of Results, Editor A. Fordy, Nonlinear Sci. Theory Appl., Manchester Univ. Press, Manchester, 1990, 97-130.

Rogers C., Shadwick W.F., Bäcklund transformations and their applications, Mathematics in Science and Engineering, Vol. 161, Academic Press, New York - London, 1982.

[4] Matveev V.B., Salle M.A., Darboux transformations and solitons, Springer Series in Nonlinear Dynamics, Springer, Berlin, 1991.

[5] Adler M., On the Bäcklund transformation for the Gel'fand-Dickey equations, Comm. Math. Phys. 80 (1981), 517-527.

[6] Levi D., Nonlinear differential difference equations as Bäcklund transformations, J. Phys. A: Math. Gen. 14 (1981), 1083-1098.

Levi D., On a new Darboux transformation for the construction of exact solutions of the Schrödinger equation, Inverse Problems 4 (1988), 165-172.

[7] Veselov A.P., What is an integrable mapping?, in What is Integrability?, Editor V.E. Zakharov, Springer Series in Nonlinear Dynamics, Springer, Berlin, 1991, 251-272.

[8] Suris Yu.B., The problem of integrable discretization: Hamiltonian approach, Progress in Mathematics, Vol. 219, Birkhäuser, Basel, 2003.

[9] Sklyanin E.K., Separation of variables. New trends, in Quantum Field Theory, Integrable Models and Beyond (Kyoto, 1994), Prog. Theor. Phys. Suppl. (1995), no. 118, 35-60, solv-int/9504001.

[10] Kuznetsov V.B., Sklyanin E.K., On Bäcklund transformations for many-body systems, J. Phys. A: Math. Gen. 31 (1998), 2241-2251, solv-int/9711010.

[11] Kuznetsov V.B., Vanhaecke P., Bäcklund transformations for finite-dimensional integrable systems: a geometric approach, J. Geom. Phys. 44 (2002), 1-40, nlin.SI/0004003.

[12] Hone A.N.W., Kuznetsov V.B., Ragnisco O., Bäcklund transformations for the sl(2) Gaudin magnet, J. Phys. A: Math. Gen. 34 (2001), 2477-2490, nlin.SI/0007041.

[13] Hikami K., Separation of variables in the BC-type Gaudin magnet, J. Phys. A: Math. Gen. 28 (1995), 4053-4061. 